\title{
Nutrients return from leaves and litterfall in a mediterranean cork oak (Quercus suber L.) forest in southwestern Spain
}

\author{
Enrique Andivia $\cdot$ Manuel Fernández $\cdot$ \\ Javier Vázquez-Piqué • Aranzazu González-Pérez • \\ Raúl Tapias
}

Published online: 21 August 2009

(C) Springer-Verlag 2009

\section{Erratum to: Eur J Forest Res}

\section{DOI 10.1007/s10342-009-0274-6}

There were errors in the Results section on the third page of the original publication, both in the text and in Table 2. In the first paragraph of the Results section ' $(10.90 \%$ of total litterfall)' should read '(55.82\% of total litterfall)' and 'acorn production $\left(2,113.68 \mathrm{~g} \mathrm{~m}^{-2}\right.$ )' should read 'acorn production $\left(87.10 \mathrm{~g} \mathrm{~m}^{-2}\right)$ '. Table 2 below shows the correct values.

The errors were due to a miscalculation and do not affect any aspect of the discussion and conclusions of the work.

Table 2 Annual values for different litterfall components in $\mathrm{g} \mathrm{m}^{-2}$ (standard error) and percentage of total litterfall of the year

\begin{tabular}{|c|c|c|c|c|c|c|}
\hline Year & Twigs & Leaves & Catkings & Acorns & Miscellaneous & Total \\
\hline March 2004-February 2005 & $\begin{array}{c}70.12(24.44) \\
14.17 \%\end{array}$ & $\begin{array}{c}274.40(55.52) \\
55.82 \%\end{array}$ & $\begin{array}{c}24.24(13.92) \\
4.93 \%\end{array}$ & $\begin{array}{c}87.10(64.97) \\
17.82 \%\end{array}$ & $\begin{array}{c}35.64(7.92) \\
7.26 \%\end{array}$ & 491.50 \\
\hline March 2005-February 2006 & $\begin{array}{c}49.88(18.84) \\
18.32 \%\end{array}$ & $\begin{array}{c}189.56(68.12) \\
69.60 \%\end{array}$ & $\begin{array}{c}14.68(15.56) \\
5.39 \%\end{array}$ & $\begin{array}{c}2.68(3.12) \\
0.98 \%\end{array}$ & $\begin{array}{c}15.56(6.84) \\
5.71 \%\end{array}$ & 272.36 \\
\hline Average & $\begin{array}{l}60.00 \\
15.71 \%\end{array}$ & $\begin{array}{l}231.96 \\
60.74 \%\end{array}$ & $\begin{array}{l}19.44 \\
5.09 \%\end{array}$ & $\begin{array}{l}44.89 \\
11.76 \%\end{array}$ & $\begin{array}{l}25.60 \\
\quad 6.70 \%\end{array}$ & 381.89 \\
\hline
\end{tabular}

The online version of the original article can be found under doi:10.1007/s10342-009-0274-6.

E. Andivia $(\bowtie) \cdot$ M. Fernández · J. Vázquez-Piqué ·

A. González-Pérez $\cdot$ R. Tapias

Department of Forest Sciences, University of Huelva,

Ctra. Palos-La Rábida, s/n. 21819 Palos de la Frontera,

Huelva, Spain

e-mail: enrique.andivia@dcaf.uhu.es 